University of Nebraska - Lincoln

DigitalCommons@University of Nebraska - Lincoln

1970

\title{
Emission of Auger Electrons Resulting from Symmetric Argon and Neon Ion-Atom Collisions
}

\author{
R. K. Cacak \\ University of Nebraska - Lincoln \\ Q. C. Kessel \\ Robert J. Van de Graaff Laboratory, High Voltage Engineering Corporation, Burlington, Massachusetts \\ M. Eugene Rudd \\ University of Nebraska - Lincoln, erudd@unl.edu
}

Follow this and additional works at: https://digitalcommons.unl.edu/physicsrudd

Part of the Physics Commons

Cacak, R. K.; Kessel, Q. C.; and Rudd, M. Eugene, "Emission of Auger Electrons Resulting from Symmetric Argon and Neon Ion-Atom Collisions" (1970). M. Eugene Rudd Publications. 56.

https://digitalcommons.unl.edu/physicsrudd/56

This Article is brought to you for free and open access by the Research Papers in Physics and Astronomy at DigitalCommons@University of Nebraska - Lincoln. It has been accepted for inclusion in M. Eugene Rudd Publications by an authorized administrator of DigitalCommons@University of Nebraska - Lincoln. 


\title{
Emission of Auger Electrons Resulting from Symmetric Argon and Neon Ion-Atom Collisions
}

\author{
R. K. Cacak ${ }^{*}$ \\ Department of Physics, University of Nebraska, Lincoln, Nebraska 68508
}

Q. C. Kessel

Robert J. Van de Graaff Laboratory, High Voltage Engineering Corporation, Burlington, Massachusetts 01803

M. E. Rudd

Department of Physics, University of Nebraska, Lincoln, Nebraska 68508

Received 17 April 1970

Cross sections for the emission of Auger electrons from excited atoms and ions produced in symmetric $\mathrm{Ar}^{+}-\mathrm{Ar}$ and $\mathrm{Ne}^{+}-\mathrm{Ne}$ collisions have been measured at ion energies from 50 to 300 $\mathrm{keV}$. A simple model is proposed to explain the dependence of the cross section on the impact energy; the predicted results agree well with the experiment. Also, the argon x-ray data of Saris and Onderdelinden are compared with the model and with the results of this experiment.

Published in Physical Review A 2, 1327 - 1331 (1970)

(C)1970 The American Physical Society. Used by permission.

URL: http://link.aps.org/doi/10.1103/PhysRevA.2.1327

DOI: 10.1103/PhysRevA.2.1327

* Present address: Department of Physics, University of Western Ontario, London, Ontario, Canada. 


\title{
Emission of Auger Electrons Resulting from Symmetric Argon and Neon Ion-Atom Collisions
}

\author{
R. K. Cacak* \\ Department of Physics, University of Nebraska, Lincoln, Nebraska 68508
}

and

Q. C. Kessel

Robert J. Van de Graaff Laboratory, High Voltage Engineering Corporation, Burlington, Massachusetts 01803

and

M. E. Rudd

Department of Physics, University of Nebraska, Lincoln, Nebraska 68508

(Received 17 April 1970)

\begin{abstract}
Cross sections for the emission of Auger electrons from excited atoms and ions produced in symmetric $\mathrm{Ar}^{+}-\mathrm{Ar}$ and $\mathrm{Ne}^{+}-\mathrm{Ne}$ collisions have been measured at ion energies from 50 to $300 \mathrm{keV}$. A simple model is proposed to explain the dependence of the cross section on the impact energy; the predicted results agree well with the experiment. Also, the argon x-ray data of Saris and Onderdelinden are compared with the model and with the results of this experiment.
\end{abstract}

\section{INTRODUCTION}

Much structure has been observed ${ }^{1,2}$ on the energy spectra of electrons resulting from energetic argon and neon ion-atom collisions. This structure appears to be superimposed upon a continuous distribution of electrons and is attributed to various autoionizing and Auger transitions within the collision partners. The purpose of this paper is to analyze the measured cross sections for exciting certain inner-shell vacancy levels by measuring the numbers of the ejected Auger electrons. Argon and neon atoms were excited by ion-atom collisions in the energy range 50-300 keV. The Auger emission cross sections have been determined by using the data of the previous paper, ${ }^{3}$ and the experimental apparatus is described elsewhere. ${ }^{4}$ Only the Auger transitions resulting from initial $K$-shell vacancies in neon and $L$-shell vacancies in argon have been considered. These are the strongest 
transitions and produce electrons with energies centered near 730 and $190 \mathrm{eV}$, respectively. The experimental results are compared with a simple, semiclassical model and also with the analogous cross section for $\mathrm{x}$-ray emission as measured by Saris and Onderdelinden. ${ }^{5}$

Structure in the argon energy-loss spectrum has been explained, ${ }^{6}$ and the emission of Auger electrons by these collisions was predicted by Fano and Lichten. ${ }^{7,8}$ According to their scheme, electrons originally in lower energy levels of the collision partners may be stranded in higher states after the collision via a "promotion" mechanism that occurs at crossings of certain molecular orbitals. These crossings generally occur at certain internuclear distances, and after the ions separate the excited atoms release most of their acquired energy via Auger and autoionizing transitions. The "diabatic" curves $^{8}$ of Lichten are estimates of the actual behavior of the molecular orbitals at intermediate internuclear distances. However, the qualitative shape of these orbitals suggests that the promotions responsible for the Auger transitions occur primarily near reasonably well-defined internuclear distances. The $4 f \sigma$ orbital of the $\mathrm{Ar}^{+}-\mathrm{Ar}$ system, for example, rises abruptly at an internuclear separation of $r_{0}=2-3 \times 10^{-9} \mathrm{~cm}$. Many promotions from the $4 f \sigma$ to other orbitals are possible and may occur within this relative narrow region. On the other hand, Lichten predicts ${ }^{8}$ that the only possible $K$ - to $L$-shell promotion in a ground-state $\mathrm{Ne}^{+}-\mathrm{Ne}$ system is the $2 p \sigma \rightarrow 2 p \pi$ transition. This crossing occurs at smaller internuclear separations than do the argon crossings, and a smaller cross section is expected.

Previous measurements ${ }^{9,10}$ have shown that the average energy loss for $\mathrm{Ar}^{+}-\mathrm{Ar}$ collisions increases sharply for those collisions whose distances of closest approach are less than $r_{0} \cong 2.5$ $\times 10^{-9} \mathrm{~cm}$. This experimental agreement with the promotion model has led us to develop a simple model to explain the dependence of the inner-shell ionization cross sections on incident ion energy.

\section{CLASSICAL CROSS-SECTION CALCULATION}

In a close encounter of two atoms, the relation between the impact parameter $p$ and the distance of closest approach $r_{0}$ depends on the type of interatomic potential. By assuming a simple screened Coulomb potential, it has been shown that ${ }^{11}$

$$
p=r_{0}\left[1-\left(b / r_{0}\right) e^{-r_{0} / a}\right]^{1 / 2},
$$

where for symmetric collision partners $b=2 Z^{2} e^{2} / E$, and the Bohr screening radius $a$ is given by $a_{0} /$ $2^{1 / 2} Z^{1 / 3}$. Here $Z$ is the atomic number of the atom and ion, $e$ is the electronic charge, $E$ is the laboratory energy of collisions, and $a_{0}$ is the first Bohr radius of hydrogen.

We assume that the excitation to Auger levels has a probability $P\left(r_{0}\right)$ of occurring during the collision, and at that impact parameter $p$ and energy $E$ the ions' internuclear distance $r_{0}$ is determined by Eq. (1). Therefore, the probability that a promotion occurs at this $r_{0}$ may be written $P\left(r_{0}(p, E)\right)$. As an approximation we now assume that the probability for producing a vacancy is $P_{c}$, a constant, for distances of closest approach less than some critical distance $r_{c}$. If the ion does not penetrate to less than $r_{c}$, the probability is assumed to be zero. This assumption is consistent with the Fano-Lichten promotion model. If the distance of closest approach of the ion-atom system is larger than the internuclear distances where promotions are possible, there will be no promotions, and the probability for an Auger transition is zero. On the other hand, if the distance of closest approach is less than $r_{c}$, there will be some finite value for $P\left(r_{0}(p, E)\right)$. In argon, a step-functional form of the probability may be justified by the fact that the probability of an ion being excited to a higher state was found ${ }^{2,10}$ to rise rapidly from zero to almost unity near internuclear distances of $\sim 2.5$ $\times 10^{-9} \mathrm{~cm}$. This is also consistent with the abrupt rise of the $4 f \sigma$ orbital from which the electrons are promoted in the Fano-Lichten model. Many crossings occur on the vertical section of the orbital within a relatively narrow range of internuclear separations, and the probability of at least one of these electrons being promoted is reasonably large. In the neon system, the $2 p \sigma \rightarrow 2 p \pi$ promotion is responsible for the $K$-shell transitions; therefore, it is reasonable to expect that the critical distance for neon is also fairly well defined.

In analogy to the definition of cross section for particular inelastic processes, ${ }^{12}$ the cross section for an Auger excitation is defined as an integral over all impact parameters, each times the probability $P\left(r_{0}(p, E)\right)$ that an Auger electron is ejected when the ion has energy $E$ and impact parameter $p$. That is, we have

$$
\sigma(E)=2 \pi \int_{0}^{\infty} P\left(r_{0}(p, E)\right) p d p
$$

In general, $P\left(r_{0}(p, E)\right)$ increases smoothly as $r_{0}$ decreases, but as a first approximation we assume a step-functional form for $P(r(p, E))$ :

$$
\begin{gathered}
P\left(r_{0}(p, E)\right)=0 \text { for } r_{0}>r_{c}, \\
P\left(r_{0}(p, E)\right)=P_{c} \text { for } r_{0} \leq r_{c} .
\end{gathered}
$$

The expression for the Auger emission cross section [Eq. (2)] now becomes

$$
\sigma(E)=\pi p_{c}^{2} P_{c}
$$

where $p_{c}$ is the impact parameter determined by 
Eq. (1) when $r_{0}=r_{c}$, and $P_{c}$ is the constant probability that an inner-shell vacancy is produced. Therefore, we have

$$
\sigma(E)=P_{c} \pi r_{c}^{2}\left[1-\left(b / r_{c}\right) e^{-r_{c} / a}\right] \text {. }
$$

The values for the probability and critical distance remain to be determined. For the argon system, the probability that either ion (or atom) of the collision is excited was determined to be $\sim 1.0$ for $r_{0} \leqq 2.5 \times 10^{-9} \mathrm{~cm}$. ${ }^{10}$ In the data to be presented, ejection of electrons from both particles was measured, ${ }^{3}$ and assuming that almost all deexcitations occur via Auger emission, the probability that an electron is ejected per collision is effectively 2. A rough estimation of this value may also be made by an examination of the molecularorbital diagram ${ }^{8}$ for argon. At each of the crossings that the $4 f \sigma$ orbital makes with the highershell orbitals, there is a finite probability for a transition. Since there are several crossings, it is reasonably likely that both electrons in the $4 f \sigma$ orbital are promoted to higher orbitals.

For the neon systems, the probability for a promotion is considerably smaller than in an argon system. The possible promotion from the $K$ to $L$ shell is sometimes blocked by full occupancy of the $2 p \pi$ orbital. Even if the $2 p \pi$ orbital has a vacancy, a promotion from the $2 p \sigma$ orbital is not guaranteed. The probability that one particle was excited to a higher state in neon was experimentally determined ${ }^{13}$ to be about $10 \%$. This excitation occurred at an internuclear distance of about $5 \times 10^{-10} \mathrm{~cm}$.

The present calculation is similar to a model presented by Fortner et al.,${ }^{14}$ but there are impor tant differences. First, a specific form of the interaction potential, a screened Coulomb potential, is assumed. This calculation can therefore predict excitation threshold for producing inner-shell vacancies as well as the behavior of the cross section near threshold. Second, the other two parameters involved, $r_{0}$ and $P$, are known empirically, and the absolute value of the cross section is calculated without a curve-fitting procedure. Finally, the present calculation is not limited by innershell excitations involving only one curve crossing. The calculation applies for the multiple-crossing case of argon as well as the single crossing of neon.

\section{RESULTS}

The cross section for Auger-electron emission was determined using the experimental results of the Paper I. ${ }^{3}$ The contribution due to the "continuum" of electrons was subtracted from the total cross section, and the region under the peak was integrated to yield the measured Auger emission cross section. This procedure was repeated for each of the ion energies considered; the results appear in Fig. 1.

Also shown are the argon-x-ray-emission results of Saris and Onderdelinden. ${ }^{5}$ The argon collision partners in their experiment are excited via argonion collisions below $100 \mathrm{keV}$. These data have been normalized to the theoretical curve at $20 \mathrm{keV}$, which raises their absolute value by a factor of $1.2 \times 10^{3}$. This value represents the reciprocal of the fluorescent yield.

The curves drawn in Fig. 1 are generated by setting $P=2.0$ and $r_{c}=2.5 \times 10^{-9} \mathrm{~cm}$ in Eq. (5) for argon and $P=0.1$ and $r_{c}=5 \times 10^{-10} \mathrm{~cm}$ for neon. The predicted shape and absolute value of the curve for argon fit well. The asymptotic form of the argon curve above $50 \mathrm{keV}$ indicates that the potential at relatively large critical distances is so weak that the impact parameter is essentially the same as the distance of closest approach.

Saris and Onderdelinden's ${ }^{5}$ results extend to lower ion energies and shown a rapid decrease below $10 \mathrm{keV}$. The $\mathrm{x}$-ray results do not show the asymptotic behavior at higher energies that the Auger cross sections exhibit, but instead seem to increase steadily as far as these data extend. This indicates a curious phenomenon. The probability of a decay of the excited argon atom (or ion) via $x$-ray emission may depend on the velocity (or energy) of the ion that excited it. This suggests the unlikely situation that the deexcitation process depends in some manner on the mode of producing

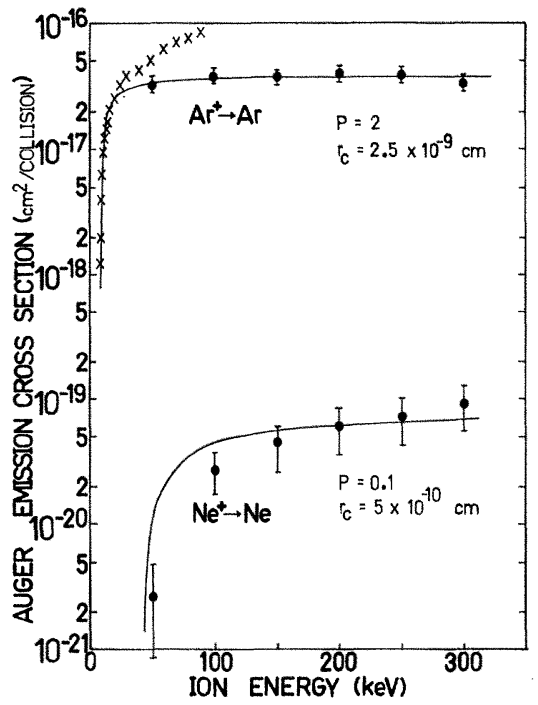

FIG. 1. Auger emission cross sections for argon and neon (circles). Crosses are the $\mathrm{x}$-ray results of Saris and Onderdelinden ${ }^{5}$ normalized at $20 \mathrm{keV}$ to the theoretical values. Solid curves are generated using Eq. (5) with the indicated parameters. 
the vacancy initially. These data also indicate that the fluorescent yield, the ratio of the $\mathrm{x}$-ray to Auger emission cross sections, depends upon the collision energy. Over the range 20-90 keV, the value that would be obtained for the fluorescent yields by heavy-ion bombardment changes by a factor of 2.5. It is evident that care must be taken in using conventional fluorescent yields to interpret heavy-ion experiments.

The neon vacancies are produced at smaller internuclear distances than are the argon vacancies. For these greater penetrations, the potential is strong enough to create a difference between the impact parameter and distance of closest approach for ions with energies as high as $150 \mathrm{keV}$. The cross section decreases rapidly below $100 \mathrm{keV}$ and appears to reach a threshold near $40 \mathrm{keV}$. Below this energy, the distance of closest approach, even for head-on collisions, is not small enough to permit the neon shells to intersect. The neon curve does not fit the data as well as in the argon case, but it is nevertheless near the correct absolute value and also predicts the threshold well.

At higher ion energies the model of Fortner et al. ${ }^{14}$ which uses the Landau-Zener theory to estimate the probability of a promotion, predicts a decrease in the $x$-ray cross section and, presumably, a decrease in the Auger-electron cross section also. Our model does not consider the velocitydependent term of the Landau-Zener theory and approaches an asymptotic value at high energies. Neither our data, Saris and Onderdelinden's, nor the carbon data of Fortner extend far enough to determine the behavior at these high velocities. Fortner's data have reached a maximum and may either decrease again or remain constant as predicted by this model. It is quite likely that the constant-probability-for-promotion arguments presented here break down at higher velocities. Higher-energy $\mathrm{x}$-ray measu rements need to be made to determine the experimental dependence of the cross sections. Auger emission measurements at ion energies higher than $300 \mathrm{keV}$ are difficult because the number of background or "continuum" of ionized electrons becomes comparable to, or larger than, the number of Auger electrons.

\section{SUMMARY}

The present results indicate that a simple model can explain the energy dependence of Auger excitation and $\mathrm{x}$-ray cross sections and thresholds for $\mathrm{x}$-ray cross sections reasonably well for some collisions. It may also be extended to include other processes that have a functional dependence on the distance of closest approach. The model does depend on the adjustment of two empirically determined parameters. However, the success of the model is demonstrated by the fact that the parameters may be evaluated from the results of independent measurements. ${ }^{2,7,13}$ Particularly, in the case of argon, these parameters can be estimated by examining the Fano-Lichten promotion model. The present results are consistent with their model for both neon and argon collisions.

\section{ACKNOWLEDGMENTS}

The authors want to express their gratitude to Dr. F. W. Saris and Dr. D. Onderdelinden for permitting them to use their data prior to publication. The interest in this problem shown by Dr. Arnold Russek is also appreciated. The experimental cross sections were the results of a thesis project of one of us (R. K. C.). We want to thank Professor Theodore Jorgensen, Jr., who served as thesis advisor, for his suggestions and advice during the project. We also wish to thank the National Science Foundation for support of the project, and the National Aeronautics and Space Administration for fellowship support.

\footnotetext{
*Present address: Department of Physics, University of Western Ontario, London, Ontario, Canada.

${ }^{1}$ M. E. Rudd, T. Jorgensen, Jr., and D. J. Volz, Phys. Rev. 151, 28 (1966); 17, 1170 (1966); C. Snoek, R. Geballe, W. F. van der Weg, P. K. Rol, and D. J. Bierman, Physica 31, 1553 (1965); G. N. Ogurtsov, I. P. Flaks, S. V. Avakyan, and N. V. Fedorenko, Zh. Eksperim. i Teor. Fiz. Pis'ma v Redaktsiyu [Soviet Phys. JETP Letters $\underline{8}, 330$ (1968)]; G. N. Ogurtsov, I. P. Flaks, and S. V. Avakyan, in Proceedings of the Sixth International Conference on the Physics of Electronic and Atomic Collisions (MIT Press, Cambridge, Mass., 1969), p. 274.

${ }^{2}$ Q. C. Kessel, M. P. McCaughey, and E. Everhart, Phys. Rev. Letters 16, 1189 (1966); 17, 1170 (1966).

${ }^{3}$ R. K. Cacak and T. Jorgensen, Jr., preceding
}

paper, Phys. Rev. A 2, 1322 (1970).

${ }^{4}$ R. K. Cacak, Ph. D. thesis, University of Nebraska, 1969 (unpublished).

${ }^{5}$ F. W. Saris and D. Onderdelinden, Physica (to be published).

${ }^{6}$ Q. C. Kessel, A. Russek, and E. Everhart, Phys.

Rev. Letters 14, 484 (1965).

${ }^{7} \mathrm{U}$. Fano and W. Lichten, Phys. Rev. Letters 14 , 627 (1965).

${ }^{8}$ W. Lichten, Phys. Rev. 164, 131 (1967).

${ }^{9}$ G. H. Morgan and E. Everhart, Phys. Rev. 128 , 667 (1962).

${ }^{10}$ Q. C. Kessel and E. Everhart, Phys. Rev. 146 , 16 (1966); E. Everhart and Q. C. Kessel, ibid. $\underline{146}$, 27 (1966).

${ }^{11}$ E. Everhart, G. Stone, and R. Carbone, Phys. Rev. 
99, 1287 (1955).

${ }^{12}$ N. F. Mott and H. S. W. Massey, The Theory of Atomic Collisions, 3rd ed. (Oxford U. P., London 1965), p. 802.

${ }^{13}$ From Ref. 2, Fig. 1 (b): The data labeled $\mathrm{Ne}^{+}-\mathrm{Ne}$ included $\mathrm{Ne}^{++}-\mathrm{Ne}$ data also. According to Lichten (Ref. 8) only one Auger electron can be produced by a $\mathrm{Ne}^{*}-\mathrm{Ne}$ collision. This being true, it was incorrect to calculate $\alpha$ for $\mathrm{Ne}^{+}-\mathrm{Ne}$ collision using the same formula as was appropriate for the $\mathrm{Ar}^{+}-\mathrm{Ar}$ Collisions. The correct maximum probability for such an excitation is approximately $10 \%$ if one assumes that only one vacancy per $\mathrm{Ne}^{*}$-Ne collision is possible.

${ }^{14}$ R. J. Fortner, B. P. Curry, R. C. Der, T. M. Kavanagh, and J. M. Kahn, Phys. Rev. 185, 164 (1969). 